\title{
Dimensão fractal e análise geomorfológica de bacias hidrográficas brasileiras
}

\author{
Fractal dimension and geomorphological analysis of \\ Brazilian watersheds
}

Cassia Brocca Caballero ${ }^{1}$, Danrlei de Menezes ${ }^{1}$, Karla Campagnolo ${ }^{1}$, Guilherme Censi ${ }^{1}$, Heron Schwarz ${ }^{1}$, Masato Kobiyama ${ }^{1}$

${ }^{1}$ Universidade Federal do Rio Grande do Sul. Porto Alegre, RS, Brasil. E-mail: cassiabrocca@gmail.com; d.menezes18@gmail.com; karlac@ufrgs.br; guilherme.censi@hotmail.com; heron.sch@gmail.com; masato.kobiyama@ufrgs.br

\begin{abstract}
Como citar: Caballero, C. B., Menezes, D., Campagnolo, K, Censi, G., Schwarz, H., \& Kobiyama, M. Dimensão fractal e análise geomorfológica de bacias hidrográficas brasileiras. Revista de Gestão de Água da América Latina, v. 17, e3, 2020. https://doi.org/10.21168/10.21168/rega.v17e3.
\end{abstract}

Resumo. A caracterização fractal da rede de drenagem de uma bacia hidrográfica surge da necessidade de se compreender a ocorrência de fenômenos hidrológicos, considerando a influência de fatores geomorfológicos. Desta forma, características físicas e fractais de uma bacia hidrográfica integram elementos importantes para análise de seu comportamento hidrológico. Assim, o objetivo deste trabalho foi estimar a dimensão fractal de sub-bacias com diferentes características pelo método de Box-Counting (Contagem de Caixas). Além disso, verificou-se a relação existente entre dimensão fractal de cursos fluviais $(d f)$ e rede de drenagem $(D f)$ e outras características da bacia hidrográfica, como comprimento do rio principal $(L)$, área $(A)$, perímetro $(P)$, densidade da rede drenagem $\left(D_{d}\right)$ e declividade média $\left(D_{\text {méd }}\right)$ da bacia. A área de estudo foi um conjunto de 6 sub-bacias, dos rios Malacara, Leão, Pavão, Faxinalzinho, Arroio Josafaz, e Forno, que pertencem à bacia hidrográfica do rio Mampituba (RS/SC). Para isso foram analisados o fator de forma $(F)$ e o coeficiente de compacidade $(K c)$ das sub-bacias e aplicado o método Box-Counting para calcular a dimensão fractal. Os valores de $F$ encontrados foram menores que 0,50 , sendo que os maiores valores foram para as sub-bacias do rio Faxinalzinho $(F=0,27)$ e Leão $(F=0,21)$ e o menor valor para a sub-bacia do Malacara $(F=0,09)$. 0 s valores de $K c$ foram todos maiores que 1,50 , indicando baixa tendência a enchentes. Diante dos valores de $d f$ e $D f$ obtidos pelo método de Box-Counting, foi possível verificar que o método se mostrou satisfatório. Observouse que existem correlações positivas significativas entre a dimensão fractal com os parâmetros largura média $(B), A$ e $P$ das bacias exploradas neste estudo. Nesse sentido, a análise da geometria fractal demonstrou ser uma metodologia viável, sendo uma ferramenta importante na avaliação geométrica de uma bacia hidrográfica.

Palavras-chave. Rede de Drenagem, Geometria Fractal, Geomorfometria de Rios.

Abstract. The fractal characterization of the catchment drainage network arises from the need to better understand how hydrological phenomena occurs, taking into account the influence of geomorphological factors. Thus, physical and fractal characteristics of a catchment integrate important elements to analyze its hydrological behavior. Thus, the objective of this work was to estimate the fractal dimension of 6 subcatchments with different characteristics by the Box-Counting method and to verify the relationship between fractal dimension of river channels $(d f)$ and drainage network $(D f)$ and other catchment characteristics such as main river length, area, perimeter, drainage density and catchment slope. The study area was a set of 6 subcatchmentsof the Malacara, Leão, Pavão, Faxinalzinho, Arroio Josafaz, and Forno rivers, which belong to the Mampituba catchment (RS/SC). The form factor $(F)$ and the compactness coefficient $(K c)$ of the catchments were analyzed and the Box-Counting method was applied to calculate the fractal dimension. The $F$ values found were less than 0.50 , and the highest values were for the Faxinalzinho $(F=0.27)$ and Leão $(F=0.21)$ catchment and the lowest value for the Malacara catchment $(F=0.09)$. The $K c$ values were all higher than 1.50 , indicating a low tendency to flooding. Given the values of $d f$ and $D f$ obtained by the Box-Counting method, it was possible to verify that this methos was satisfactory. It is observed that there are significant positive correlations

Recebido: Março 19, 2020. Revisado: Maio 06, 2020. Aceito: Maio 11, 2020 
between the fractal dimension with average basin width, area and perimeter of the catchments in this study. In this sense, the fractal geometry analysis proved to be a viable methodology, being an important tool in the geometric assessment of a catchment.

Keywords. Drainage Network; Fractal Geometry; River Geomorphometry.

\section{INTRODUÇÃO}

A bacia hidrográfica é utilizada como a unidade geomorfológica fundamental, em consequência de suas características físicas que governam o fluxo superficial da água. Essas características desempenham um papel importante no ciclo hidrológico, estando ligadas diretamente ao comportamento hidrológico da rede de drenagem (Marques et al., 2012). Silva et al. (2016) destacam ainda área, forma e topografia como sendo características morfológicas que definem o comportamento hidrológico de uma bacia hidrográfica. Desta forma, a Geomorfologia, ciência que estuda as formas de relevo, é de extrema importância na hidrologia.

A rede hidrográfica de uma bacia, sendo uma característica geomorfológica da bacia e estando diretamente ligada aos mecanismos que formam o relevo, é um elemento formador da paisagem de uma região, influenciando nos processos que envolvem sua construção e diversidade (Carneiro et al., 2010). Segundo Christofoletti (1980), redes de drenagem são definidas como unidades geomórficas correspondentes à área drenada por um rio ou sistema fluvial, e os canais localizados dentro dessa bacia estão conectados e organizam-se em redes complexas. Dessa maneira, as diversas características dinâmicas no escoamento nas ravinas, canais e tributários, componentes de redes hidrográficas, são responsáveis pelas qualidades atribuídas aos processos fluviais.

Os canais das redes fluviais são resultantes da complexa interação entre os processos climáticos e geológicos, refletindo nas principais propriedades de evolução das paisagens (Jati \& Silva, 2017). Os fatores que determinam os mecanismos hídricos são extremamente variáveis em sua distribuição espacial e temporal, o que torna a representação matemática destes fenômenos muito complexos. Dessa maneira, a busca por métodos que permitam o mapeamento mais detalhado possível da complexidade do sistema de drenagem é essencial (Moussa \& Bocquillon, 1993).

Na tentativa de descrever quantitativamente as redes de drenagem, Horton (1945) introduziu um modelo hierárquico de ordenação de rede de canais, denominado Leis de Horton, que foi posteriormente modificado por Strahler (1952). Estas Leis de Horton vêm sendo tradicionalmente utilizadas para análise morfométrica em bacias hidrográficas (Vestena \& Kobiyama, 2010). Porém, nos últimos anos, juntamente com o avanço do uso do sensoriamento remoto, uma nova abordagem tem sido utilizada para compreender as formas irregulares das redes de drenagem: a análise fractal (Marques \& Gomes, 2014). 0 uso da geometria fractal é uma alternativa para identificar quantitativamente as características de uma bacia hidrográfica e permitir, dessa maneira, conhecer as relações existentes entre os processos hidrológicos (Rodríguez-Iturbe \& Rinaldo, 1997).

0 conceito de geometria fractal foi criado por Mandelbrot (1983) e trata-se do ramo da matemática que estuda as propriedades e comportamento dos fractais. Segundo Pereira et al. (2016), este conceito ajuda a descrever padrões de distribuição de estruturas naturais, ou seja, de formas irregulares que possuem autossimilaridade e/ou autoafinidade.

A dimensão fractal tornou-se o modelo matemático mais eficiente para descrever paisagens reais, pois parece capturar a essência da topografia superficial da Terra de maneira que outros atributos geomorfológicos não o fazem (Xu et al., 1993). Tarboton et al. (1988) confirmam que os fractais fornecem uma estrutura matemática para o tratamento de formas irregulares e complexas, pois exibem padrões semelhantes em diversas escalas (Figura 1). Assis et al. (2008) descrevem que as principais propriedades que caracterizam os fractais são a autossemelhança, a complexidade infinita e a sua dimensão, onde a autossemelhança é identificada quando uma porção pode ser vista como a réplica do seu total, em uma escala menor. A complexidade infinita refere-se ao fato de o processo de geração de um fractal ser recursivo, e a dimensão de um fractal, que não precisa ser necessariamente um valor inteiro, representa o grau de ocupação da estrutura no espaço. 


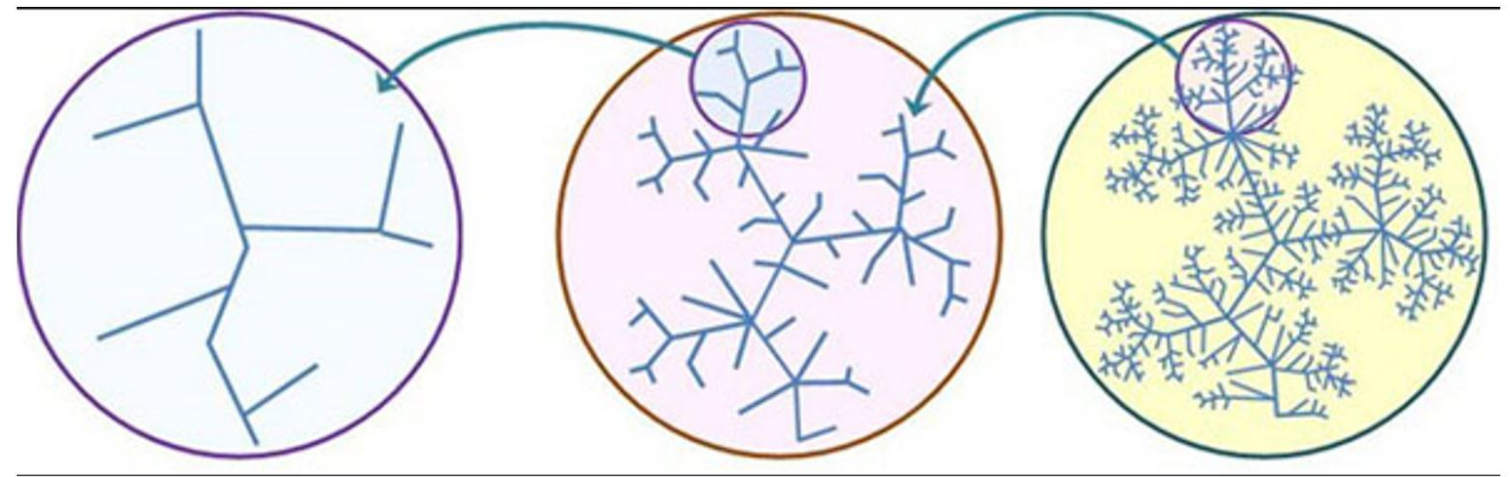

Figura 1. A autossemelhança dos fractais significa que cada parte em escala menor é semelhante à parte inicial. Fonte: Tunas et al. (2016).

Nesse sentido, a geometria fractal é útil para descrever padrões irregulares e fragmentados encontrados na natureza. Conforme Schüller et al. (2001), o litoral é um exemplo que foi bastante analisado na dimensão fractal pois, ao medir o comprimento de uma costa em um mapa, os pesquisadores descobriram que o comprimento total depende da escala selecionada, assim quanto menor a unidade de medida, mais longa é a costa. Esse fenômeno ocorre devido à natureza fractal do litoral. Assim, pode-se dizer que um objeto com menor dimensão fractal é menos complexo do que um objeto com maior dimensão fractal (Vestena \& Kobiyama, 2010). Assis et al. (2008) confirmam que um fractal é um objeto que apresenta invariância na sua forma à medida em que a escala é alterada, mantendo-se a sua estrutura idêntica à original.

Ainda, a descrição da complexidade da paisagem natural através da linguagem da geometria fractal pode abrir caminho para se entender a interação de influências climáticas e tectônicas no desenvolvimento de paisagens, considerando as relações de escala (Chase, 1992), ou fornecer informações que podem ser utilizadas em diferentes níveis de planejamento de recursos hídricos (Vestena \& Kobiyama, 2010).

0 uso da geometria fractal na hidrologia e na geomorfologia vem avançando há décadas. Um exemplo pode ser visto em Rodríguez-Iturbe \& Rinaldo (1997), os quais demonstraram que uma bacia é caracterizada pela multifractalidade. Isso significa que os valores de dimensão fractal para canais $(d f)$ e para rede inteira $(D f)$ são diferentes. Esses valores foram primeiramente estabelecidos com o uso das Leis de Horton, a partir de equações.

Diversos trabalhos aplicaram o conceito de dimensão fractal na análise da rede de drenagem de bacias hidrográficas, correlacionando com a aplicação das leis de Horton, destacando-se os trabalhos de La Barbera \& Rosso (1987), Tarboton et al. (1988), Rosso et al. (1991), Veltri et al. (1996), Agnese et al. (1996), Tarboton (1996), Roth et al. (1996), Kobiyama \& Bueno Junior (2002), Bueno Junior (2002), Silveira (2006), Vestena \& Kobiyama (2010) e Tavares et al. (2017). Estes trabalhos reconhecem que as redes fluviais são estruturas fractais que apresentam propriedades de autossimilaridade ou autoafinidade, sob uma série significativa de escalas. Segundo Silveira (2006) esses resultados impactam significativamente estudos sobre a evolução de bacia hidrográfica e sobre determinação das respostas em redes fluviais.

Desta forma, características físicas e fractais de uma bacia hidrográfica integram elementos importantes para análise de seu comportamento hidrológico. Isso porque, estabelecendo-se relações e comparações entre eles e dados hidrológicos, colabora-se para o entendimento de diversos itens relacionados à dinâmica ambiental em regiões onde processos hidrológicos são variados e complexos e também onde dados existentes são insuficientes. Desta maneira, o objetivo principal deste trabalho foi estimar a dimensão fractal de 6 sub-bacias com diferentes características (declividade, densidade de drenagem) pelo método de Box-Counting (Contagem de Caixas) e verificar a relação existente entre dimensão fractal e outras características da bacia hidrográfica como declividade e densidade de drenagem. 


\section{MATERIAL E MÉTODOS}

\section{1 Área de Estudo}

A área de estudo compreende um conjunto de seis sub-bacias, dos rios Malacara, Leão, Pavão, Faxinalzinho, Arroio Josafaz, e Forno, as quais pertencem à bacia hidrográfica do rio Mampituba, que divide os estados do Rio Grande do Sul e Santa Catarina (Figura 2).
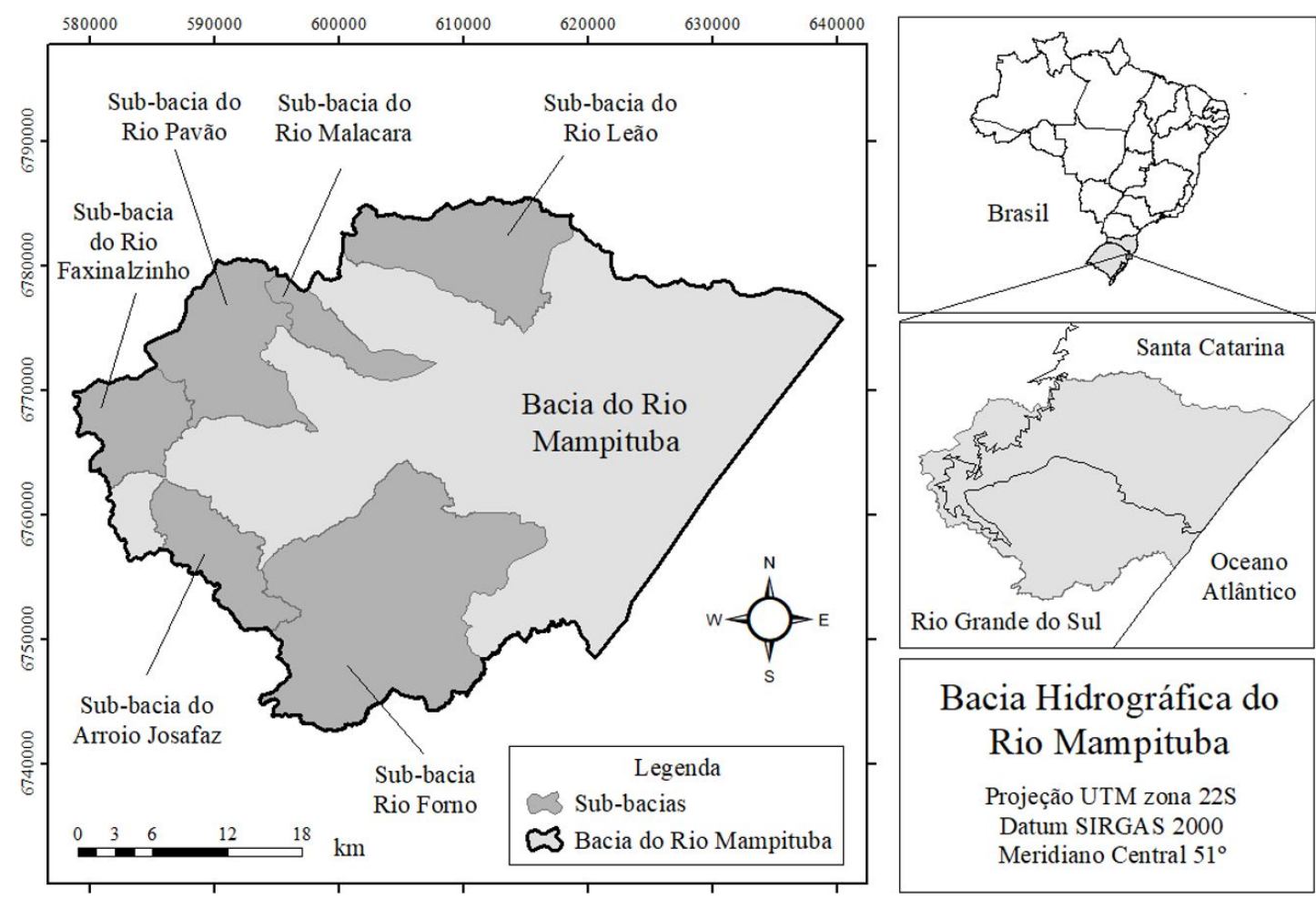

Figura 2. Localização das sub-bacias do rio Mampituba analisadas.

A região das escarpas da serra pertence à Unidade Geomorfológica Serra Geral, com relevo forte ondulado a montanhoso de altitudes variando entre 100 e $1.000 \mathrm{~m}$ acima do nível do mar, expondo sequências de derrames da Formação Serra Geral e de rochas areníticas da Formação Botucatu (Instituto Brasileiro do Meio Ambiente e dos Recursos Naturais Renováveis, 2004). Ainda, nestes locais os processos evolutivos formaram o principal atrativo paisagístico da região, os cânions. Entre as várias feições geomorfológicas do relevo nesta região destacam-se os Cânions do Itaimbezinho, Malacara, Fortaleza e Índios Coroados, que devido às belezas naturais são bastante explorados pelo turismo local.

Em relação à vegetação, no planalto dos campos gerais a principal formação florestal existente corresponde à Floresta Ombrófila Mista, também denominada de Floresta de Araucárias que, segundo Scheibe et al. (2010), também é a vegetação predominante na região de Praia Grande. A vegetação original está bastante alterada, principalmente nas áreas planas, onde foi substituída por cultivos agrícolas (milho, feijão, banana e fumo). Nas maiores altitudes, a vegetação está preservada, constituída principalmente por floresta nativa secundária e florestamentos de pinus e eucalipto (Sehnem et al., 2015).

O clima da região segundo a classificação de Köppen é Cf (Mesotérmico Úmido, sem estação seca), e inclui dois subtipos: Cfa (Clima Subtropical Mesotérmico Úmido) com verões quentes e invernos frios, próximo ao litoral; e Cfb (Clima Temperado Mesotérmico Úmido) com inverno frio e verão ameno, nas partes mais elevadas da Serra (Moreno, 1961; Pandolfo et al., 2002). A precipitação média anual da região é de $1.500 \mathrm{~mm}$ e evapotranspiração média anual de $900 \mathrm{~mm}$ (Pandolfo et al., 2002). A intensidade máxima da chuva com duração de 5 minutos é de $217 \mathrm{~mm} \cdot \mathrm{h}^{-1}$, com período de retorno de 5 anos (Back, Bonetti, 2014). Esses aspectos, juntamente com a mudança brusca de altitude entre 
as partes mais altas da serra e a planície, favorecem a ocorrência de inundações bruscas, escorregamentos e fluxo de detritos.

\subsection{Caracterização das sub-bacias}

Os parâmetros fator de forma $(F)$ e coeficiente de compacidade $\left(K_{c}\right)$ são índices utilizados para definir a forma de uma bacia hidrográfica. $0 \mathrm{~F}$ compara a bacia a um retângulo, sendo que quanto maior o seu valor, maior a potencialidade de ocorrência de picos de enchentes elevados. Em relação ao $K_{c}$, este compara a bacia hidrográfica a um círculo $\left(K_{c} \geq 1\right)$, sendo que quanto maior o valor deste parâmetro, mais distante de um círculo é a forma da bacia hidrográfica. Quanto mais a bacia hidrográfica se aproxima de um círculo, maior a concentração de deflúvio no exutório, propiciando uma tendência para enchentes. 0 parâmetro densidade de drenagem $\left(D_{d}\right)$ consiste em relacionar o comprimento total dos canais e a área da bacia hidrográfica, e esta relacionado com o tempo gasto para o escoamento superficial da bacia atingir o exutório.

As características físicas da bacia como a largura média da bacia $(B)$, o $F$, o $K_{c}$ e a $D_{d}$ foram obtidos pelas Equações 1, 2, 3 e 4, respectivamente,

$$
\begin{aligned}
& B=\frac{A}{L} \\
& F=\frac{A}{L^{2}} \\
& K_{c}=0,282 \times \frac{P}{\sqrt{A}} \\
& D_{d}=\frac{\sum L_{i}}{A}
\end{aligned}
$$

onde $A$ é a área da bacia $\left(\mathrm{km}^{2}\right) ; L$ é o comprimento do rio principal $(\mathrm{km}) ; P$ é o perímetro da bacia $(\mathrm{km})$; e $\Sigma L_{i}$ é o comprimento total dos canais da bacia $(\mathrm{km})$.

\subsection{Dimensão Fractal}

A delimitação da bacia hidrográfica do Rio Mampituba e das sub-bacias selecionadas para o estudo da dimensão fractal (DF) foi baseada no modelo digital do terreno (MDT) proveniente dos dados da Shuttle Radar Topographic Mission (SRTM) disponibilizados pela United States Geological Survey (USGS) (https://earthexplorer.usgs.gov/). O software ArcGIS® 10.3 foi utilizado em conjunto com as extensões Spatial Analyst e Arc Hydro Tools para realizar a delimitação automática das bacias hidrográficas. Ao final do procedimento, correções foram aplicadas sobre os produtos gerados pelo software por meio de interpretação visual. Os dados de hidrografia na escala 1:50.000 foram obtidos a partir da base cartográfica vetorial contínua do Rio Grande do Sul (Hasenack \& Weber, 2010).

Para calcular a $D F$ foi aplicada a metodologia desenvolvida por Goodchild (1982) de Box-Counting. Para empregar o método é necessário produzir uma malha quadriculada projetada sobre a figura, no caso a rede de drenagem. Ou seja, o método consiste em quebrar um conjunto de dados, tal como um objeto ou imagem, em pequenos pedaços ou caixas. Dessa forma, foram projetadas malhas quadriculadas sobre a rede de drenagem das bacias em estudo. Com esta malha sobre a rede de drenagem, foram contadas as quadrículas que interceptaram as linhas da figura. Conhecendo-se o tamanho de caixa (d) e o número de caixas $N(d)$, a $D F$ pode ser calculada pela Equação 5 (Tunas et al., 2016):

$D F=\lim _{d \rightarrow 0} \frac{\log N(d)}{\log 1 / d}$

onde $N(d)$ é o número de caixas cobrindo a rede de drenagem e $d$ é o tamanho da caixa. $N(d)$ é recalculado até o tamanho da caixa se aproximar de zero e, então, é plotado na forma de um gráfico log-log, onde revela-se uma relação linear, cuja declividade da linha de tendência ou declividade da reta representa a dimensão fractal. Em um gráfico log-log, plotam-se os valores de $d$ e $N(d)$, e obtém-se uma relação linear 
entre $\log d$ e $\log N(d)$ com inclinação de $-k_{o}$, conforme representado na Equação 5 , onde $\mu$ é o coeficiente angular da reta.

$\log N(d)=\log \mu-k_{. o} \log d$

A dimensão fractal é o valor representado por $k_{o}$. Para cálculo da $D F$ sobre a rede de drenagem, foram consideradas oito malhas quadriculadas com diferentes tamanhos de caixas, considerando a faixa de valores recomendada por Tarboton et al. (1996). Tarboton et al. (1988) relatam que para o cálculo da dimensão fractal de cursos fluviais $(d f)$ devem ser usados valores de $d$ entre 15 a $125 \mathrm{~m}$. Já para a dimensão fractal da rede de drenagem $(D f)$ de uma bacia hidrográfica, foi definido que $d$ seria de 250 a $1.000 \mathrm{~m}$. Sendo assim, os valores de $d$ adotados foram ajustados para a escala das bacias em estudo, de 1:50.000, sendo utilizados $d$ correspondentes a 0,5 mm, 1,0 mm, 1,5 mm e 2,0 mm, 3,0 mm para o cálculo de $d f$; e para o cálculo de $D f$, foram utilizadas malhas quadriculadas com tamanho de caixa $d$ de $6,0 \mathrm{~mm}, 10,0 \mathrm{~mm}, 20,0 \mathrm{~mm}$. Na escala real, estes tamanhos equivalem a 25, 50, 75, 100, 150, 300, 500 e $1000 \mathrm{~m}$, respectivamente.

Após a obtenção desses valores, objetivando verificar o grau de correlação entre $D f$ e $d f$ com as características físicas da bacia $\left(K_{c}, F, L, A, P, D_{m e ́ d} D_{d}\right)$, foi realizada uma análise de regressão linear simples. Para isto, foi utilizado o coeficiente de determinação $R^{2}$, que varia de $0 \leq R^{2} \leq 1$, sendo que quanto maior o $\mathrm{R}^{2}$, mais explicativo é o modelo, ou seja, melhor ele se ajusta à amostra. A partir disso, é possível estabelecer as relações entre essas variáveis analisadas.

\section{RESULTADOS}

\subsection{Caracterização das sub-bacias}

Na Tabela 1 são apresentados os resultados obtidos quanto aos parâmetros físicos das bacias analisadas. É possível destacar as diferentes áreas e declividades obtidas. A declividade média $\left(D_{\text {méd }}\right)$ da

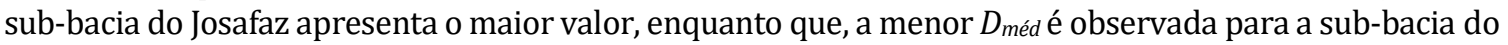
Leão, que por sua vez, apresenta uma maior área.

Tabela 1. Características físicas das bacias analisadas.

\begin{tabular}{|c|c|c|c|c|c|c|c|c|}
\hline Sub-bacia & $\begin{array}{c}A \\
\left(\mathbf{k m}^{2}\right)\end{array}$ & $P(\mathbf{k m})$ & $L(\mathrm{~km})$ & $\begin{array}{c}B \\
(\mathbf{k m})\end{array}$ & $\boldsymbol{F}$ & $K c$ & $\begin{array}{c}\text { Declividade } \\
\text { Média }\left(D_{m e ́ d}\right) \\
(\%)\end{array}$ & $\begin{array}{c}\text { Densidade da } \\
\text { rede de } \\
\text { drenagem }\left(D_{d}\right) \\
\left(\mathrm{km}^{2} \mathbf{k m}^{-2}\right)\end{array}$ \\
\hline Faxinalzinho & 58,52 & 44,84 & 14,67 & 3,99 & 0,27 & 1,65 & 29,3 & 1,28 \\
\hline Forno & 284,03 & 118,81 & 42,31 & 6,71 & 0,16 & 1,99 & 18,8 & 0,88 \\
\hline Josafaz & 63,30 & 63,28 & 20,17 & 3,14 & 0,16 & 2,24 & 39,5 & 1,46 \\
\hline Leão & 110,54 & 70,09 & 22,73 & 4,86 & 0,21 & 1,88 & 18,7 & 1,09 \\
\hline Malacara & 34,96 & 42,25 & 19,99 & 1,75 & 0,09 & 2,02 & 25,3 & 1,12 \\
\hline Pavão & 102,98 & 64,46 & 29,07 & 3,54 & 0,12 & 1,79 & 22,4 & 1,34 \\
\hline
\end{tabular}

\subsection{Dimensão fractal utilizando o método de Box-Caunting.}

Na Figura 3 é demonstrada a aplicação do método de Box-counting para as sub-bacias em estudo, utilizando o tamanho de quadrícula de 20,0 $\mathrm{mm}$ na escala 1:50.000. Já na Tabela 2 são apresentados os dados de $d, N(d), \log d$ e $\log N(d)$ para a bacia do rio Faxinalzinho, utilizados para a plotagem do gráfico apresentado na Figura 4 e o cálculo das dimensões fractais $d f$ e $D f$. Dessa forma, os resultados dos cálculos de $d f$ e $D f$ para todas as bacias de estudo são apresentados na Tabela 3. 


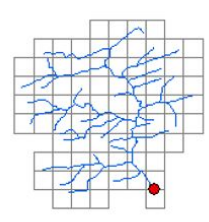

Sub-bacia do Rio Faxinalzinho

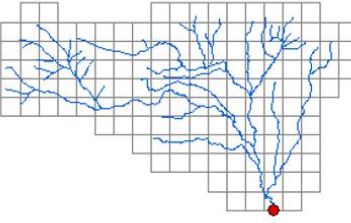

Sub-bacia do Rio Leão

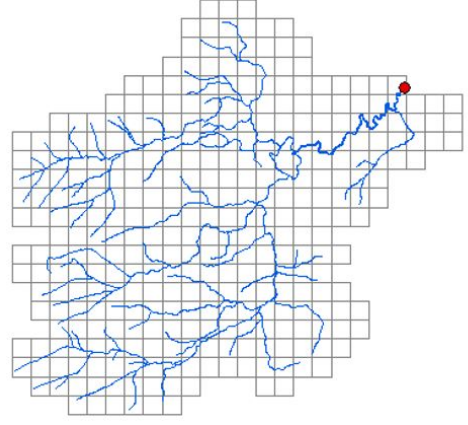

Sub-bacia do

Rio F orno

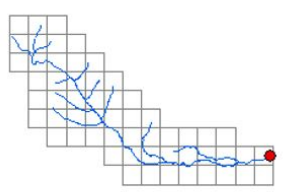

Sub-bacia do

Rio Malacara

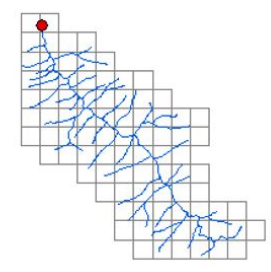

Sub-bacia do Arroio Josafaz

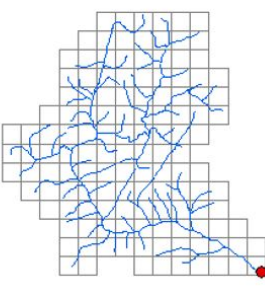

Sub-bacia do Rio Pavão

Figura 3. Contagem das caixas pelo método de Box-Counting utilizando o tamanho de quadrícula de 1000 metros (20,0 mm na escala 1:50.000).

Tabela 2. Dados necessários para obtenção da dimensão fractal para canais $(d f)$ e da rede de drenagem $(D f)$ para a bacia do rio Faxinalzinho.

\begin{tabular}{|c|c|c|c|c|}
\hline & $d(\mathrm{~mm})$ & $N(d)$ & $\log d$ & $\log N(d)$ \\
\hline \multirow{4}{*}{$d f$} & 0,5 & 3769 & $-0,301$ & 3,576 \\
\hline & 1,0 & 1867 & 0,000 & 3,271 \\
\hline & 1,5 & 1248 & 0,176 & 3,096 \\
\hline & 2,0 & 919 & 0,301 & 2,963 \\
\hline \multirow{4}{*}{$D f$} & 3,0 & 2760 & 0,477 & 3,441 \\
\hline & 6,0 & 725 & 0,778 & 2,860 \\
\hline & 10,0 & 280 & 1,000 & 2,447 \\
\hline & 20,0 & 78 & 1,301 & 1,892 \\
\hline
\end{tabular}

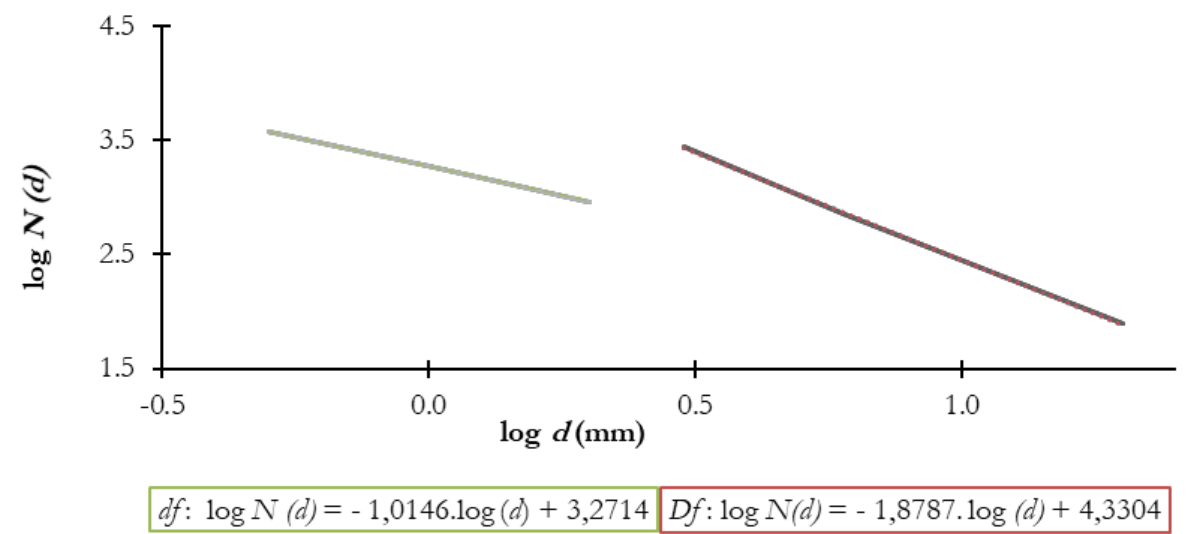

Figura 4. Gráfico $\log d x \log N(d)$ para determinação de $d f$ e $D f$ para a bacia do rio Faxinalzinho. 
Tabela 3. Dimensão fractal das sub-bacias do rio Mampituba.

\begin{tabular}{c|c|c}
\hline \multirow{2}{*}{ Sub-bacias } & \multicolumn{2}{c}{ Dimensão fractal } \\
\cline { 2 - 3 } & 1,16 & Df \\
\hline Faxinalzinho & 1,29 & 1,75 \\
\hline Forno & 1,20 & 1,91 \\
\hline Josafaz & 1,21 & 1,81 \\
\hline Leão & 1,10 & 1,68 \\
\hline Malacara & 1,22 & 1,80 \\
\hline Pavão & &
\end{tabular}

\subsection{Relações entre a dimensão fractal e características físicas das bacias}

Relacionando os valores obtidos na Tabela 3 com as características físicas das bacias (Tabela 1) através do coeficiente de determinação $\mathrm{R}^{2}$, obteve-se uma alta correlação com as características morfométricas básicas das bacias $(A, P, L, B)$, não se estendendo, porém, às características compostas, como $F$ e $K c$, conforme pode-se observar na Figura 5. As melhores correlações de $D f$ com as características morfométricas foram para $B$, que apresentou o melhor $\mathrm{R}^{2}$ (igual a 0,8916 ), seguido da $A\left(\mathrm{R}^{2}=0,8839\right)$ e do $P\left(\mathrm{R}^{2}=0,8795\right)$. Para o $d f$, as melhores correlações foram para $P\left(\mathrm{R}^{2}=0,8328\right)$, $B\left(\mathrm{R}^{2}=0,8024\right)$ e para $A\left(\mathrm{R}^{2}=0,7701\right)$. Sendo assim, essas características fornecem estimativas a priori dos resultados que envolvem a geometria fractal. Nesse caso, os parâmetros mais sensíveis no cálculo da geometria fractal para bacias montanhosas, como é o caso deste estudo, são $B, A$ e $P$.
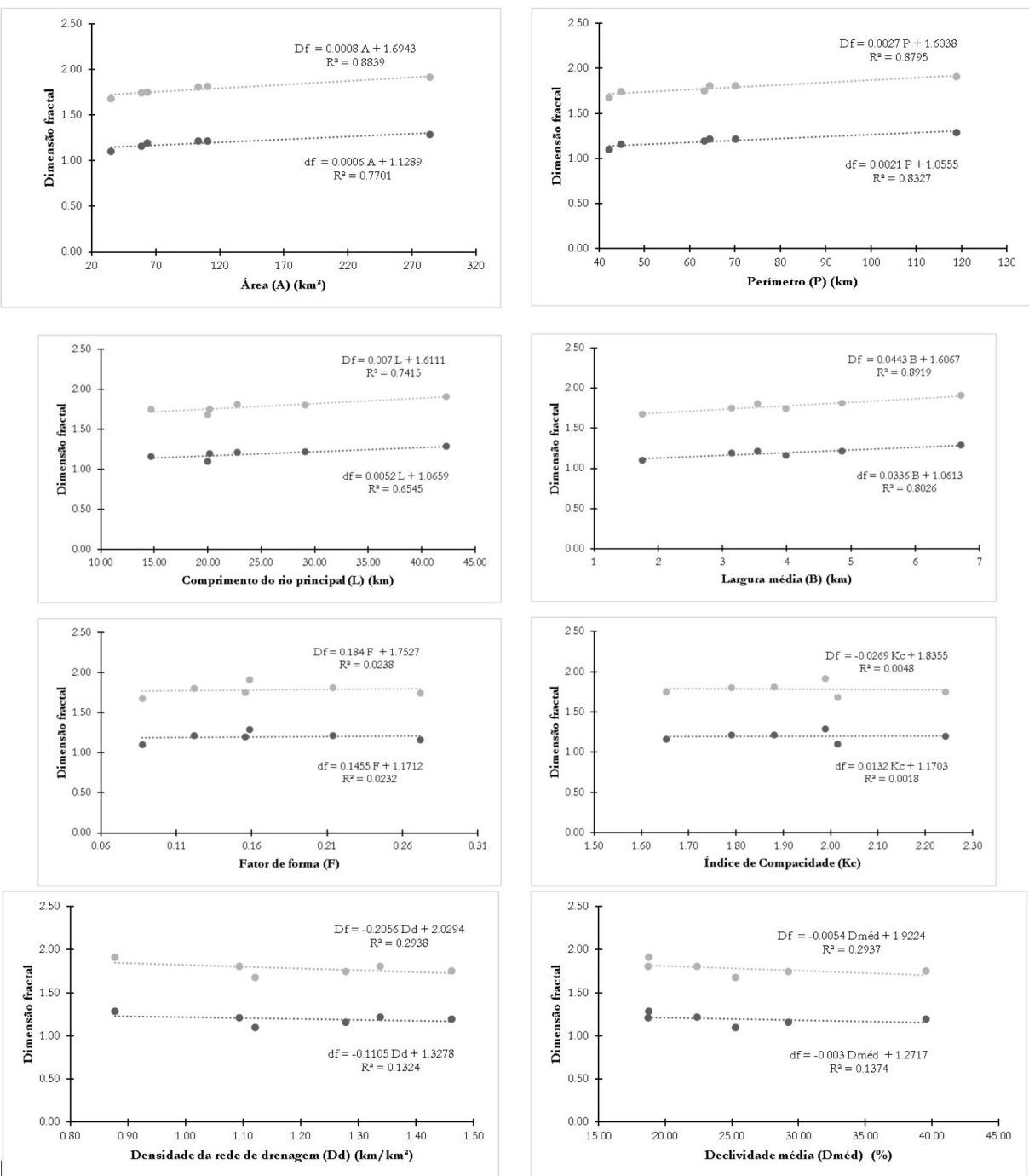

Figura 5. Relações entre as dimensões fractais e as características físicas da bacia. 


\section{DISCUSSÃO}

Analisando os resultados apresentados na Tabela 1, observa-se que as áreas de cada sub-bacia diferiram significativamente, sendo a menor delas a sub-bacia do Malacara, com área de $34,96 \mathrm{~km}^{2}$ - e a maior delas a sub-bacia do Forno, com área de $284,03 \mathrm{~km}^{2}$. Dessa maneira, assim também os valores de $F$ e de $K_{c}$ variaram. Maiores valores de $F$ indicam a tendência da bacia a ter picos de enchentes, sendo que valores menores ou iguais a 0,50 indicam uma baixa tendência de enchentes na bacia (Gerber et al., 2018). Nesse sentido, nas sub-bacias estudadas, todos os valores de $F$ foram menores que 0,50 , em que os maiores valores foram encontrados para as sub-bacias do rio Faxinalzinho $(F=0,27)$ e Leão $(F=0,21)$ e o menor valor para a sub-bacia do Malacara $(F=0,09)$.

$0 K_{c}$ compara a forma da bacia a um círculo, em que bacias hidrográficas cuja forma se aproxima à de um círculo tendem a proporcionar a conversão do escoamento superficial para um trecho pequeno do rio principal; assim, quanto mais próximo a 1 for este índice maior a potencialidade de picos de enchentes (Oliveira et al., 2010). No geral, os valores de $K_{c}$ foram todos maiores que 1,50, indicando baixa tendência a enchentes (Gerber et al., 2018), sendo os mais altos para as sub-bacias Josafaz $\left(K_{c}=2,24\right)$ e Malacara $\left(K_{c}=2,02\right)$, indicando uma tendência de forma alongada nessas sub-bacias. Esse fato aumenta o tempo de concentração e diminui a probabilidade de enchentes em comparação com uma bacia de mesma área, mas com formato mais próximo de uma circunferência.

0 índice de $D_{d}$ é importante a ser considerado visto que reflete a influência da geologia, topografia, vegetação e solos de uma bacia hidrográfica, estando diretamente relacionado com o tempo gasto para o escoamento superficial da bacia. Desse modo, quanto maior for a $D_{d}$, maior será a capacidade da bacia de fazer escoamentos rápidos no exutório. Para as bacias em estudo, conforme pode-se observa na Tabela 1, o índice de $D_{d}$ encontra-se entre 0,5 e $1,5 \mathrm{~km} / \mathrm{km}^{2}$, o que indica que estas bacias possuem uma drenagem regular, sendo que para uma bacia ser considerada bem drenada, o índice $D_{d}$ deve ser superior a 3,5.

Considerando os resultados obtidos, pode-se classificar as sub-bacias em estudo como de baixa suscetibilidade a enchentes em condições normais de precipitação, constatado em virtude de o $K_{c}$ apresentar o valor afastado da unidade e o $F$ exibir um valor baixo. Deste modo, pode-se considerar que as bacias em estudo não possuem forma circular e, sim, tendência alongada (Oliveira et al., 2010). Porém, apesar dessas características alongadas das sub-bacias, episódios de ocorrência de enchentes e movimentos de massa na região têm ocorrido com frequência nos últimos anos (Melo et al., 2018; Schwarz et al., 2018; Zambrano, 2017; Zanandrea et al., 2018), principalmente devido à alta declividade das bacias, perceptível pela grande variação de altitude do local.

Um estudo realizado na Bacia Hidrográfica do rio Debossan (Nova Friburgo-RJ), região de alta declividade com alta cobertura florestal, demonstrou uma área pouco suscetível a enchentes em condições normais de precipitação, encontrando um valor de $K_{c}$ afastado da unidade $(1,58)$ e também um valor baixo para o $F(0,33)$ (Cardoso et al., 2006). Já Teodoro et al. (2007), analisando a microbacia do córrego Marivan, em Araraquara, São Paulo, predominantemente urbana (70\% da bacia urbanizada) encontraram um $F$ de 1,07 e o $K_{c}$ de 0,91 , indicando que o perímetro da bacia aproxima-se a um círculo, favorecendo os processos de inundação (cheias rápidas).

Gerber et al. (2018), analisando as características morfométricas da Bacia Hidrográfica do Rio Itajaí, encontraram $K_{c}$ afastado da unidade $(1,51)$ e um baixo $F(0,43)$, indicando que a bacia não possui formato semelhante ao de uma circunferência, correspondendo, portanto, a uma bacia alongada. No entanto, apesar das características dessa bacia não indicarem propensão a enchentes, os autores detalham que as regiões do médio e alto vale do Rio Itajaí têm ocorrências frequentes de enchentes. Os autores atribuem essa divergência ao fato das pesquisas considerarem a caracterização morfométrica da bacia geral e não realizarem um estudo específico das microbacias que apresentam suscetibilidade à ocorrência de enchentes.

Nessa perspectiva, a análise da dimensão fractal é importante para compreender a dinâmica dos processos hidrológicos e a evolução das redes de drenagem em bacias hidrográficas (Vestena \& Kobiyama, 2010). Na Tabela 3 foi apresentado os valores da dimensão fractal encontrados neste estudo para as sub-bacias do rio Mampituba, sendo o valor mínimo encontrado 1,10 e o máximo 1,29. Estes valores encontram-se entre 1,0 e 2,0, faixa aceitável para este tipo de análise, podendo ser considerados, portanto, como fractais (Kobiyama et al., 2001). Além disso, os valores de $D f$ foram maiores que $d f$, o que é o esperado para este tipo de análise, demonstrando resultados satisfatórios (Tavares et al., 2017). Ainda, os valores encontrados neste estudo vão de acordo com os encontrados na literatura neste tipo de análise (Silveira, 2006; Vestena \& Kobiyama, 2010; Marques et al., 2012; Marques \& Gomes, 2014; Tavares et al., 2017). 
Valores de $d f$ próximo a 1 mostram que os canais individuais, apresentam uma pequena sinuosidade sobre a superfície. Esse resultado corrobora com o fato que os canais das sub-bacias analisadas são desenvolvidos sobre uma alta declividade, não possibilitando a formação de meandros pelos canais fluviais. Além disso, os altos valores de $D f(\geq 1,80)$ podem evidenciar uma complexidade na rede de drenagem das sub-bacias em análise, o que se aplica diretamente à realidade das sub-bacias analisadas, visto que são bacias localizadas em terreno sinuoso. Tavares et al. (2017), aplicando o método de Box-Couting para cálculo dos valores de dimensão fractal para a rede de drenagem da bacia hidrográfica do rio Maquiné (RS), encontraram $d f$ de 1,008 e $D f$ de 1,975. Esses valores são similiares aos encontrados neste estudo, onde ambas as áreas estudadas apresentam grande variação de altimetria (de 3 a 975 metros).

Os valores da dimensão fractal obtidos neste estudo demostraram-se coerentes com a teoria fractal, comprovando assim a existência das propriedades de autossimilaridade e de multiescalonamento nas redes de drenagem das seis sub-bacias analisadas. A dimensão fractal demonstra a regularidade escalar da rede de drenagem de uma bacia hidrográfica. 0 conjunto espacial manifesta uma relação escalar entre o número de seus elementos constituintes e a sua classe de mensuração (tamanho, densidade e intensidade), o que promove a eliminação do problema da escala de representação dos mapas.

\section{CONCLUSÕES}

A aplicação da geometria fractal nas sub-bacias analisadas indicou que seus cursos fluviais e a rede de drenagem podem ser considerados fractais. 0 método Box-Counting, metodologia aplicada neste estudo, demonstrou ser uma metodologia viável e de fácil aplicação. Dessa forma, sua aplicação demonstra ser uma ferramenta útil na avaliação geométrica de uma bacia hidrográfica, pois permite uma avaliação complementar, principalmente no tocante a pesquisas de redes de drenagem e sua forma.

A análise dessas características pela ótica fractal permite avaliar aspectos relacionados à drenagem, relevo e geologia de uma bacia hidrográfica, podendo levar a uma compreensão de diversas questões associadas à dinâmica ambiental local, fornece subsídios para a gestão de recursos hídricos e desastres hidrológicos. Sugere-se que novos estudos que abordem a geometria fractal avaliem e comparem sistemas de drenagem diferenciados, visando estabelecer as dimensões fractais que os representem.

\section{AGRADECIMENTOS}

O presente trabalho foi realizado com apoio da Coordenação de Aperfeiçoamento de Pessoal de Nível Superior - Brasil (CAPES) - Código de Financiamento 001 e Programa Pró-Recursos Hídricos, Chamada $n^{\circ} 16 / 201$.

\section{REFERÊNCIAS BIBLIOGRÁFICAS}

Agnese, C., D’Asaro, F., Grossi, G., \& Rosso, R. (1996). Scaling properties of topologically random channel networks. Journal of Hydrology (Amsterdam), 187, 183-193.

Assis, T.A., Miranda, J.G.V., Mota, F.B., Andrade, R.F.S., Castilho, C.M.C. (2008). Geometria fractal: propriedades e características de fractais ideais. Revista Brasileira de Ensino de Física, 30(2), 2304.

Back, A. J., \& Bonetti, A. V. (2014). Chuva de projeto para instalações prediais de águas pluviais de Santa Catarina. Revista Brasileira de Recursos Hídricos, 19(4), 260-267.

Bueno Junior, J. (2002). Análise morfométrica da bacia do Rio Marumbi-PR, baseada nas leis de Horton e na Geometria Fractal (Monografia). UFPR, Curitiba.

Cardoso, C.A., Dias, H.C.T., Soares, C.P.B., Martins, S.V. (2006). Caracterização morfométrica da bacia hidrográfica do rio Debossan, Nova Friburgo-RJ. Árvore, 30(2), 241-248.

Carneiro, P. R. F., Cardoso, A. L., Zampronio, G. B., \& Martingil, M. C. (2010). A gestão integrada de recursos hídricos e do uso do solo em bacias urbano-metropolitanas: o controle de inundações na bacia dos Rios Iguaçu/Sarapuí, na Baixada Fluminense. Ambiente \& Sociedade, 13(1), 29-49.

Chase, C. G. (1992). Fluvial landsculpting and the fractal dimension of topography. Geomorphology, 5, 39-57.

Christofoletti, A. (1980). Geomorfologia. São Paulo: Ed. Edgard Blucher. 
Gerber, D., Pertille, C. T., Vieira, F. S., Corrêa, B. J. S., \& Souza, C. F. (2018). Caracterização morfométrica da Bacia Hidrográfica do Rio Itajaí - Santa Catarina. Acta Biológica Catarinense, 5(1), 72-83.

Goodchild, M. F. (1982). The fractal Brownian processes as a terrain simulation model. Modeling and Simulation (Vol. 13, pp. 1133-1137). Pittsburgh.

Hasenack, H., \& Weber, E. (Orgs.) (2010). Base cartográfica vetorial contínua do Rio Grande do Sul - escala 1:50.000 (No. 3, Série Geoprocessamento). Porto Alegre: UFRGS Centro de Ecologia. 1 DVD-ROM.

Horton, R. (1945). Erosional development of streams and their drainage basins: a hydrophysical approach to quantitative morphology. Geological Society of America Bulletin, 56(3), 275-370.

Instituto Brasileiro do Meio Ambiente e dos Recursos Naturais Renováveis - IBAMA. (2004). Plano de Manejo dos Parques Aparados da Serra e Serra Geral. Brasília: IBAMA.

Jati, D. A., \& Silva, J. T. (2017). Estudos geo-hidrológicos da bacia do rio Curuá-Una, Santarém, Pará: aplicação do modelo hidrológico de grandes bacias (MGB-IPH). Revista Brasileira de Geografia Física, 10(4), 1296-1311.

Kobiyama, M., \& Bueno Junior, J. (2002). Multifractal characteristics of drainage network of Marumbi river watershed, Paraná state. In Anais do XXXI Congresso Brasileiro de Engenharia Agrícola (pp. 432-435).

Kobiyama, M., Bueno Junior, J., Fruet, D., \& Santos, I. (2001). Análise do hidrograma das bacias dos rios Nhundiaquara e Marumbi, PR: estudo preliminar com geometria fractal. In Anais do XIV Simpósio Brasileiro de Recursos Hídricos.

La Barbera, P., \& Rosso, R. (1987). Fractal geometry of river networks. Water Resources Research, 27(3), 381-387.

Mandelbrot, B. B. (1983). The fractal geometry of nature (468p.). New York: W.H. Freeman and Company.

Marques, L. S., \& Gomes, M. H. R. (2014). Análise fractal do ribeirão marmelo. In Anais do XII Simpósio de Recursos Hídricos do Nordeste (8 p.).

Marques, L., Bortoni, S. F., \& Gomes, M. H. R. (2012). Análise da Bacia Hidrográfica do Ribeirão Marmelo sob o Enfoque da Geometria Fractal. In Anais do XI Simpósio de Recursos Hídricos do Nordeste (13p.).

Melo, C. M., Kobiyama, M., \& Michel, G. P. (2018). Importância da caracterização geotécnica no mapeamento de áreas susceptíveis a escorregamentos no uso do modelo Shalstab. In Anais do I Encontro Nacional de Desastres (8 p.). Porto Alegre: ABRHidro.

Moreno, J. A. (1961). Clima do Rio Grande do Sul (42 p.). Porto Alegre: Secretaria da Agricultura.

Moussa, R., \& Bocquillon, C. (1993). Morphologie fractale du réseau hydrographique. Hydrological Sciences Journal, 38(3), 187-201.

Oliveira, P. T. S., Sobrinho, T. A., Steffen, J. L., \& Rodrigues, D. B. B. (2010). Caracterização morfométrica de bacias hidrográficas através de dados SRTM. Revista Brasileira de Engenharia Agrícola e Ambiental, 14(8), 819-825.

Pandolfo, C., Braga, H. J., da Silva Jr, V. P., Massignam, A. M., Pereira, E. S., Thomé, V. M. R., \& Valci, F. V. (2002). Atlas climatológico digital do Estado de Santa Catarina. Florianópolis: EPAGRI.

Pereira, M. A. F., Campo, G. F. N., Kobiyama, M., \& Castro, N. M. R. (2016). Regionalização com geometria hidráulica e fractal: estudo de caso com hidrograma unitário instantâneo geomorfológico. Revista Brasileira de Recursos Hídricos, 21(2), 347-359.

Rodríguez-Iturbe, I., \& Rinaldo, A. (1997). Fractal river basins: chance and self-organization (547 p.). Cambridge: Cambridge University Press.

Rosso, R., Bacchi, B., \& La Barbera, P. (1991). Fractal relation of mainstream length to catchment area in river networks. Water Resources Research, 27(3), 381-387.

Roth, G., Labarbera, P., \& Greco, M. (1996). On the description of the basin effective drainage structure. Journal of Hydrology (Amsterdam), (187), 119-135.

Scheibe, L.F., Buss, M.D., Furtado, S.M.A. (2010). Atlas ambiental da Bacia do rio Araranguá: Santa Catarina - Brasil (64 p.). Florianópolis: UFSC, Cidade Futura.

Schüller, D. J., Rao, A. R., \& Jeong, G. D. (2001). Fractal characteristics of dense stream networks. Journal of Hydrology (Amsterdam), 243(1-2), 1-16.

Schwarz, H., Cardozo, G. L., \& Michel, G. P. (2018). Análise da identidade espectral evolutiva da vegetação em cicatrizes de escorregamentos na bacia do rio mascarada RS. In Anais do Encontro Nacional de Desastres. Porto Alegre: ABRHidro.

Sehnem, M., Rodrigues, G.P., Camargo, G.I.L., Corseuil, C.W., D’Aquino, C.A. (2015). Análise morfometria de bacias hidrográficas sujeitas a inundações na região sul de Santa Catarina - Brasil. In Anais do XXI Simpósio Brasileiro 
de Recursos Hídricos. Recuperado em 27 de setembro de 2017, de http://www.evolvedoc.com.br/sbrh/detalhes-938_analise-da-morfometria-de-bacias-hidrograficas-sujeitasa-inundacoes-na-regiao-sul-de-santa-catarina-brasil

Silva, R. M., Costa, A. L. R., Silva, G. M. S., Souza, C., Teixeira, N. C., \& Silva, A. R. C. B. (2016). Análise das características morfométricas da bacia hidrográfica rio das Pitas. In Anais do 6o Simpósio de Geotecnologias no Pantanal (Cuiabá, MT) (15 p.). Embrapa Informática Agropecuária, INPE.

Silveira, N. F. Q. (2006). Análise fractal de bacias hidrográficas de região de encosta e região de planalto com base em cartas topográficas e em fotografias aéreas (Tese de doutorado). Universidade Federal de Santa Catarina, Florianópolis.

Strahler, A. N. (1952). Hypsometric (area-altitude) analysis of erosional topography. Bulletin. Geological Society of America, 63(1), 117-114.

Tarboton, D. G. (1996). Fractal river networks, Horton's laws and Tokunaga cyclicity. Journal of Hydrology (Amsterdam), 187(1-2), 105-117.

Tarboton, D. G., Bras, R. L., \& Rodriguez-Iturbe, I. (1988). The fractal nature of river networks. Water Resources Research, 24(8), 1317-1322.

Tavares, T. G. A., Silva, D. F., \& Kobiyama, M. (2017). Geometria fractal da bacia hidrográfica do Rio Maquiné/RS. In Anais do XXII Simpósio Brasileiro de Recursos Hídricos.

Teodoro, V. L. J., Teixeira, D., Costa, D. J. L., \& Fuller, B. B. (2007). 0 conceito de bacia hidrográfica e a importância da caracterização morfométrica para o entendimento da dinâmica ambiental local. Revista UNIARA, 20, 137-156.

Tunas, I. G., Anwar, N., \& Lasminto, U. (2016). Fractal characteristic analysis of watershed as variable of synthetic unit hydrograph model. The Open Civil Engineering Journal, 10, 706-718.

Veltri, M., Veltri, M., \& Maiolo, M. (1996). On the fractal description of natural channel networks. structure. Journal of Hydrology (Amsterdam), (187), 137-144.

Vestena, L. R., \& Kobiyama, M. (2010). A geometria fractal da rede de drenagem da bacia hidrográfica do Caeté, Alfredo Wagner-SC. Revista Árvore, 34(4), 661-668.

Xu, T., Moore, I. D., \& Gallant, J. C. (1993). Fractals, fractal dimensions and landscapes - a review. Geomorphology, 8, 245-262.

Zambrano, F. C. (2017). Avaliação do perigo de inundações bruscas por meio de modelagem hidrogeomorfologica: estudo de caso, bacia do arroio Forromeco-RS (Dissertação de mestrado). Programa de Pós-graduação em Recursos Hídricos e Saneamento Ambiental, Instituto de Pesquisas Hidráulicas, Universidade Federal do Rio Grande do Sul, Porto Alegre.

Zanandrea, F., Cardozo, G. L., Michel, G. P., \& Kobiyama, M. (2018). Parâmetros geomorfológicos para avaliação pósdesastre de escorregamentos estudo de caso da bacia hidrográfica do rio mascarada RS. In Anais do I Encontro Nacional de Desastres (8 p.). Porto Alegre: ABRHidro.

\section{Contribuição dos Autores}

Cassia Brocca Caballero, Danrlei Menezes e Karla Campagnolo foram responsáveis pela escrita do artigo, análise dos dados e revisão dos resultados.

Guilherme Censi e Heron Schwarz foram responsáveis pela aplicação da metodologia, geração dos resultados e revisão da escrita.

Masato Kobiyama é o professor orientador deste trabalho, sendo responsável pela revisão de todas as etapas da elaboração deste artigo. 